\title{
Wavelength Monitoring with Semiconductor Laser Amplifiers
}

\author{
A. Hurtado*, A. González-Marcos, J.A. Martín-Pereda \\ E.T.S.I. Telecomunicación, Universidad Politécnica de Madrid \\ c/ Ciudad Universitaria s/n, 28040-Madrid (Spain)
}

\begin{abstract}
The semiconductor laser diodes that are typically used in applications of optical communications, when working as amplifiers, present under certain conditions optical bistability, which is characterized by abruptly switching between two different output states and an associated hysteresis cycle. This bistable behavior is strongly dependent on the frequency detuning between the frequency of the external" optical signal that is injected into the semiconductor laser amplifier and its own emission frequency. This means that small changes in the wavelength of an optical signal applied to a laser amplifier causes relevant changes in the characteristics of its transfer function in terms of the power requirements to achieve bistability and the width of the hysteresis. This strong dependence in the working characteristics of semiconductor laser amplifiers on frequency detuning suggest the use of this kind of devices in optical sensing applications for optical communications, such as the detection of shifts in the emission wavelength of a laser, or detect possible interference between adjacent channels in DWDM (Dense Wavelength Division Multiplexing) optical communication networks.
\end{abstract}

Keywords: Optical Bistability, Wavelength Monitoring, Semiconductor Laser Amplifier, DWDM.

\section{INTRODUCTION}

Optical bistability in laser diode amplifiers has been in the last years an intensive research topic due to the huge potential applications of this devices in different fields of technology, such as optical computing and optical communications[1-4] and for their practical advantages of laser amplifiers: the presence of gain, fast response, low optical power requirements to achieve bistability etc. Concretely, in optical computing, this nonlinear behavior exhibited by the laser amplifier leads to the possibility of using this kind of devices as basic components in the developing of logic gates, that will later conform photonic circuits for optical signal processing or optical storage. In the other hand, in optical communications this devices could be employed in optical switching applications, optical signal regeneration, optical head packet processing etc.

The more intensively studied mode of operation of laser diode amplifiers has been the transmission mode of operation, which is characterized by the appearance of anticlockwise (or S-Shaped) bistable loops. More recently the reflective mode of operation has been also studied, having been demonstrated the existence of new forms of bistability apart from the anticlockwise bistable loop, such as the X-shaped and the clockwise bistable loop[1]. This new shapes of bistability suggest new possibilities for practical applications of this kind of devices. Anyway, the optical bistability either in reflection or in transmission, is very strongly dependent on the bias current applied to the laser amplifier, and also on the frequency detuning existing between the external optical signal and the resonant frequency mode of the laser[1][2], showing that very small changes on this parameter causes very strong variations in the input-output optical power relation of the laser amplifier.

What we suggest in this work is, making use of this strong frequency dependence of the optical bistability in laser amplifiers, the possible application of this devices in the development of structures, capable to detect very small shifts on the wavelength of a particular optical signal. This wavelength shifts could eventually cause failures in optical communication systems that are very sensitive to variations on the wavelength, such as the DWDM (Dense Wavelength Division Multiplexing) optical communication networks, where the wavelength spacing between channels is very small. Then, possible shifts in the wavelength could lead to interference between two adjacent channels, with an expensive cost in the performance of the system. Thus, an effective approach for wavelength monitoring is required.

\footnotetext{
" hurtado@tfo.upm.es; phone: +34915495762 ext.425
} 


\section{THEORY}

The physical phenomenon that causes the optical bistability in laser amplifiers is the dispersive nonlinearity, characterized by the existence of a resonator filled with a medium whose refractive index depends on the incident optical power. Increments in the optical power injected into a laser makes its resonant frequency to move towards longer wavelengths, due to the variation of the refractive index. This means that when an optical signal, detuned to the long wavelength side of the resonant frequency mode of the laser is injected into it, will cause, as a consequence of the variation of the refractive index of the active medium, the positive wavelength shift of the resonant emission frequency of the laser. Once a threshold is achieved the optical bistability can be observed. For a more detailed explanation of the optical bistability in laser amplifiers see for example[3].

In fig. 1 are represented the optical input-output power relations calculated from the equations of the model proposed by Adams [1-2] for different values of frequency detuning and for both modes of operation: transmission and reflection. A constant value of the unsaturated gain coefficient $\mathrm{g}_{0}$ is kept, and the magnitude of the frequency detuning $\phi_{0}$, is varied from $-0.15 \pi$ to $-0.4 \pi$, being $\pi$ the complete laser mode spacing. The laser parameters chosen are: cavity length $L=350 \mu \mathrm{m}$, internal loss coefficient, $\alpha=5000 \mathrm{l} / \mathrm{m}$, material linewidth enhancement factor, $b=6.9$, confinement factor $\Gamma=0.5$, front and back laser facet reflectivities are considered equals with a value of, $R_{I}=R_{2}=R=0.3$. From fig. 1 can be appreciated the strong dependence on the frequency detuning of the laser amplifier performance, either in transmission or reflection. In both cases, higher values of detuning correspond to higher values of optical input power to achieve bistability and wider biestable loops[1][4]. In the transmission mode of operation the input-output power relations keep a constant anticlockwise bistable loop shape in any case[4]. In the other hand, in the reflective mode of operation, not only the power needed for bistability or the bistable loop width vary with increments on the initial frequency detuning but also the characteristic shape of the bistable loop changes as the detuning increases from an anticlockwise bistable loop to a clockwise bistable loop, with an intermediate X-shape shape state[1].
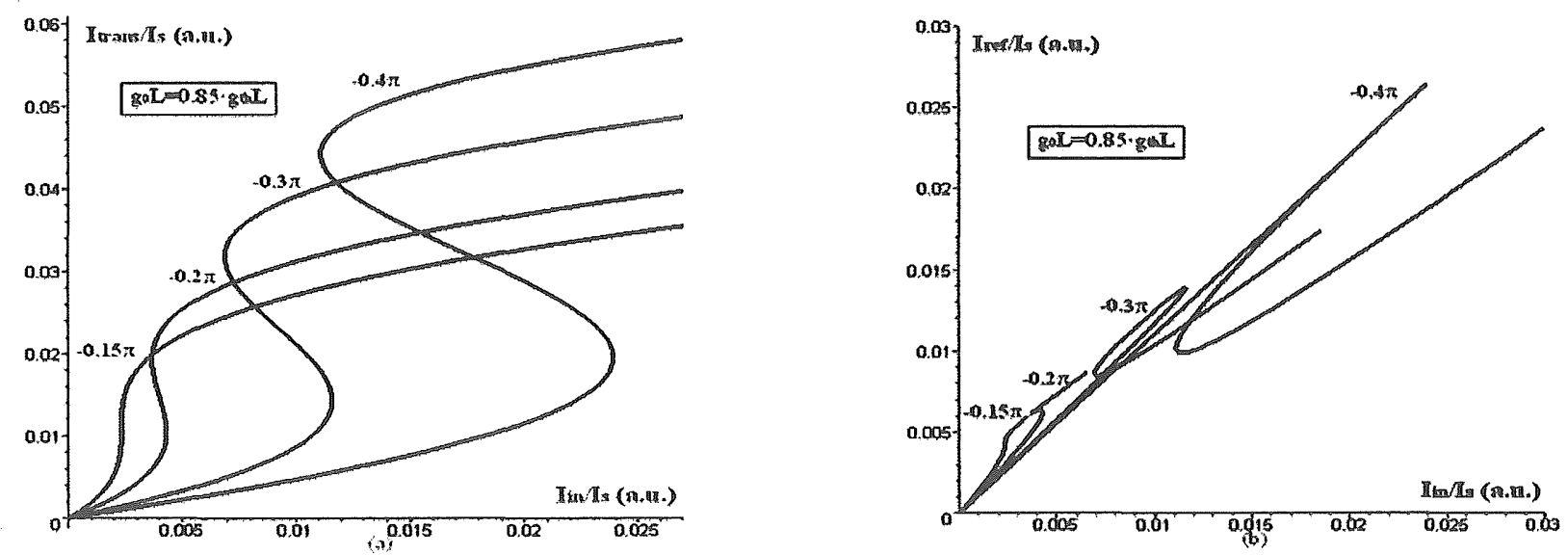

Fig. 1. Input-Output optical power relations of a Fabry-Perot semiconductor laser amplifier for different values of initial frequency detuning, (a) in the transmission mode of operation (b) and in the reflective mode of operation.

\section{STRUCTURE OF THE WAVELENGTH SENSOR}

The structure proposed for the wavelength shift sensor based in laser amplifiers is shown schematically in fig. 2. Two lasers are used, the first one being operated in reflection and the second one in transmission, where the input signal of the second laser is directly the output signal of the first one. This structure, whose transfer function has been analyzed theoretically[5], has been previously proposed by the authors for the construction of optical logic cells in the $1550 \mathrm{~nm}$ wavelength range[6]. For this previous application, a constant an unique frequency was used for the external optical signal entering to the device. Nevertheless, as this structure is based on semiconductor laser amplifiers, which exhibit an strong dependence on the frequency detuning, it is expected that the input-output power response of the complete structure will also vary when the frequency of the external signal is modified. A verification of the frequency dependence 
is shown on figs. 3(a-d), where the optical input-output relation is represented for four different values of the frequency of the optical signal entering to the device, with a frequency difference among them of only a few Ghz. An increase in frequency makes the transfer function of the device to suffer a 'compression' effect, as the power requirements to achieve bistability for the lasers are lowered due to the reduction of the relative frequency detuning. In the opposite side an external signal frequency decrease, leads to a higher frequency detuning, and as the power needed for bistability is increased, the transfer function suffers a 'relaxation' effect. As a consequence of this behavior, if a constant optical power signal is injected to this device, the amount of optical power obtained at the output of the device will depend on the frequency of the signal.

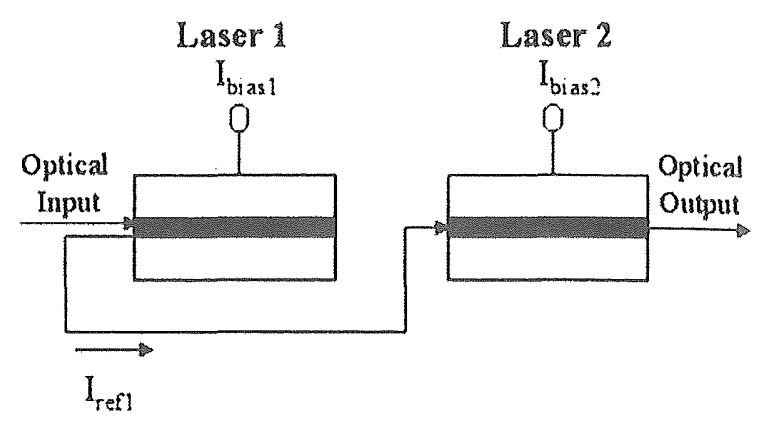

Fig. 2. Schematic Structure of the semiconductor laser wavelength shift sensor.
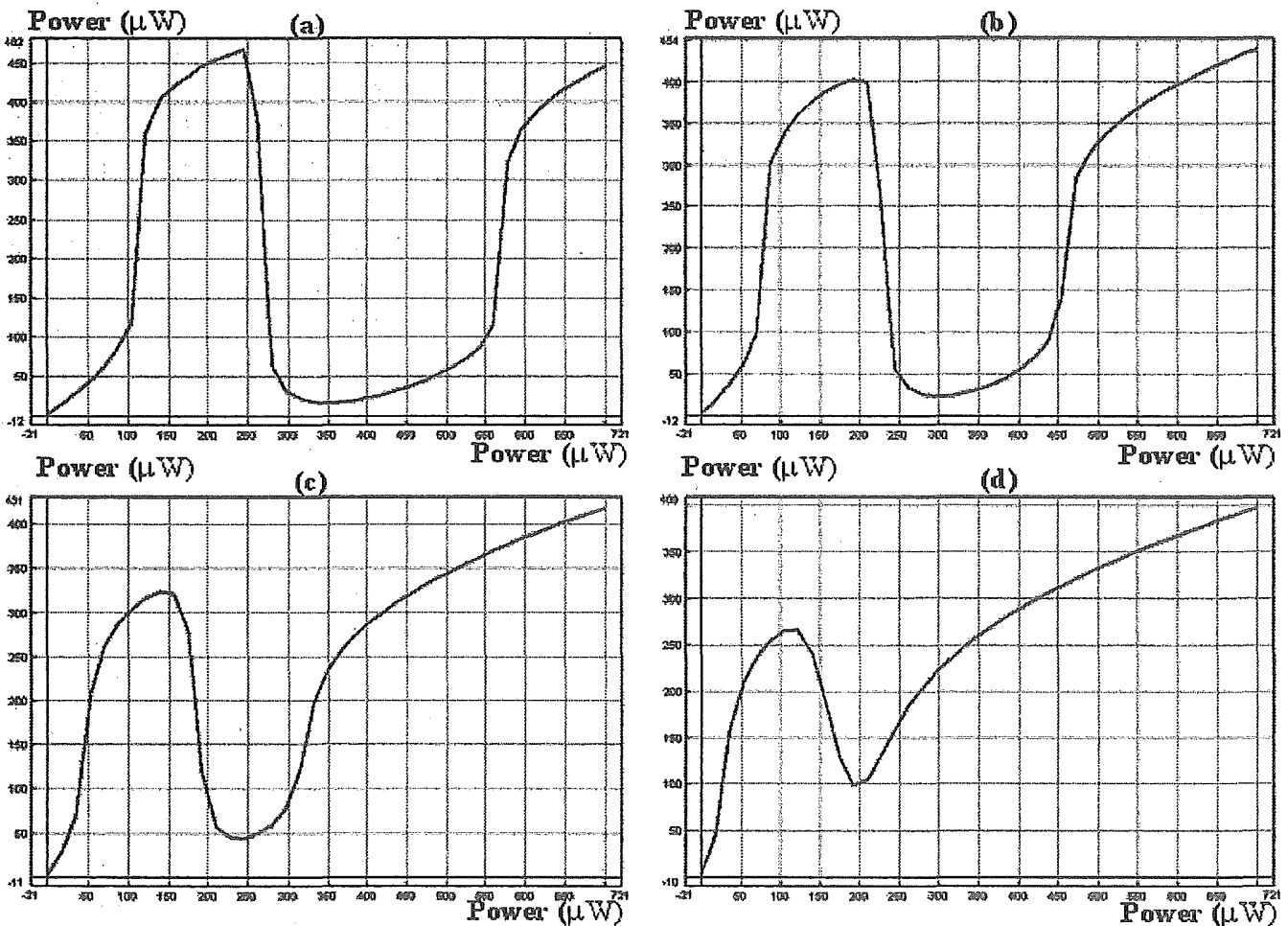

Fig. 3. Optical Input-Output power relations of the structure for (a) 193.095Thz, (b) 193.098Thz, (c) 193.102Thz and (d) $193.105 \mathrm{Thz}$.

Fig. 4. shows the output power of the device when a $0.25 \mathrm{~mW}$ constant power optical signal is injected into it, as a function of its frequency, in a $40 \mathrm{Ghz}$ wide interval around $193.1 \mathrm{Thz}(1552.52 \mathrm{~nm})$. The graphical representation obtained for that frequency interval shows a 'mountain-valley' like shape, where different zones of frequency supply different values of constant output power. The main conclusion given by this representation is, that when a constant input power signal is injected into the device, adjacent narrow intervals of the signal frequency respond with different constant output power values, separated by abruptly change zones. This behavior suggest the application of this kinds of devices as detectors of possible wavelength shifts of the emission frequency of the lasers that conform the individual multiplexed channels in Dense Wavelength Division Multiplexed (DWDM) optical telecommunication networks, by sending an optical alarm signal when a shift in wavelength is produced, and also by the amplitude of this alarm signal the direction of the shift in wavelength could be determined.

DWDM optical systems are characterized by the massive multiplexation of a huge number of channels in the $1550 \mathrm{~nm}$ wavelength range (each one of them situated in a particular wavelength), that are propagated together along a same optical fiber, incrementing considerably the fiber systems capacity[7]. Typical separation between adjacent channels in 
the $1550 \mathrm{~nm}$ wavelength range are $100 \mathrm{Ghz}, 50 \mathrm{Ghz}, 25 \mathrm{Ghz}$, and in the next future, this channel separation magnitudes are supposed to be eventually reduced. As a consequence of the very small value of the channel separation, the DWDM optical transmitters that conform the individual channels, should be very stabilized in wavelength to avoid eventual frequency shifts. That could cause a particular channel to interfere with an adjacent one producing possible system failures. As the channel separation keeps reducing, more sophisticated transmission elements are needed, and will be also needed devices that could detect instantly a displacement in the particular wavelength of a channel sending an alarm signal. This alarm signal would indicate the direction of a wavelength displacement and the measures that should be applied in order to correct it avoiding possible interferences two individual channels, what could eventually cause the communications over that two channels to be lost or deteriorated.

Considering a narrow channel separation DWDM system (i.e. 25Ghz), the sensor device proposed in this work could be used to detect instantly wavelength shifts on the emission frequency of the laser that conforms a particular channel of the system situated at $193.1 \mathrm{Thz}(1552.52 \mathrm{~nm})$ using only $250 \mu \mathrm{W}$ of the total power emitted by the optical source. The sensor is configured to detect shifts of the emitted signal of $5 \mathrm{Ghz}$ on each direction. The working mechanism of the sensor can be seen in fig. 4: if the emission frequency of the optical source is situated within the permitted frequency interval, the output power obtained from the sensor is lower than $50 \mathrm{microW}$, when the frequency is reduced, this magnitude is kept until the output signal switches suddenly, sending an alarm signal with an amplitude higher than 450 microW indicating a low frequency band shift. In the opposite side, if the frequency moves towards higher frequencies above a certain threshold, an abrupt change in the output power of the sensor will configure an alarm signal of amplitude equal to $175 \mu \mathrm{W}$, indicating a high frequency band shift in the emission wavelength on the laser that conforms the DWDM channel. A further analysis allows identifying sensor power input tolerance for difference wavelength range of the signal to sense.

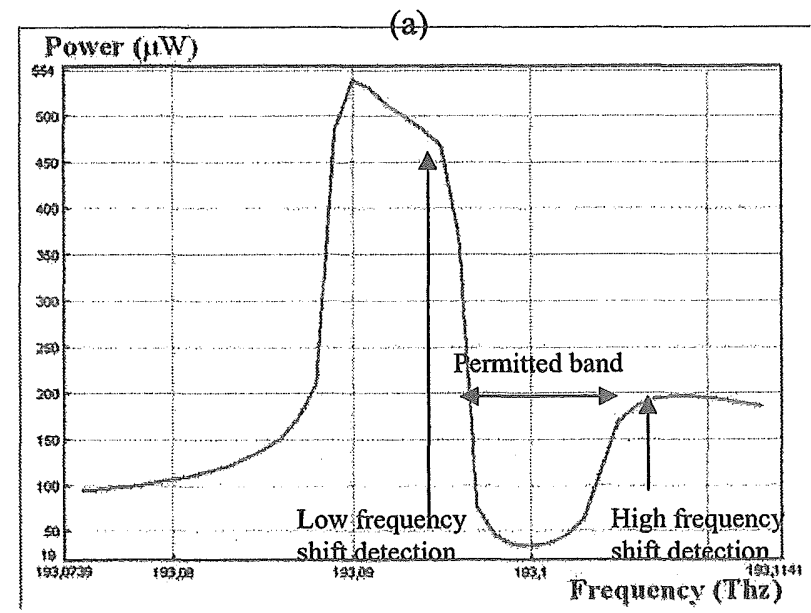

(b)

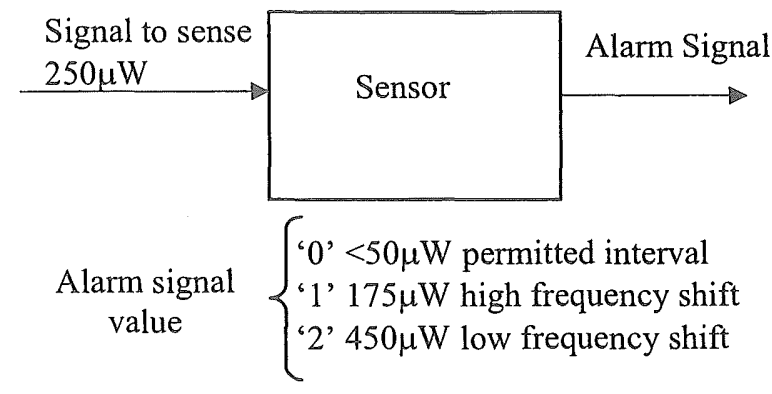

Fig. 4. (a) Optical output power as a function of the frequency. $0.25 \mathrm{~mW}$ input signal amplitude, (b) block-diagram of the sensor.

\section{REFERENCES}

1. P. Pakdeevanich and M. J. Adams, 'Measurements and Modeling of Reflective Bistability in 1.55- $\mu m$ Laser Diode Amplifiers', IEEE J. Quantum Electron., Vol. 35, NO. 12, pp. 1894-1903, Dec. 1999.

2. M. J. Adams, 'Time-Dependent analysis of active and passive optical bistability in semiconductors', Proc. IEE, vol. 132, pt. J., pp.343-348, Dec. 1985.

3. Hitoshi Kawaguchi, Bistabilities and Nonlinearities in Laser Diodes, Artech House 1994.

4. A. Hurtado, A. González-Marcos, J.A. Martín-Pereda, 'Análisis de la desintonización de Frecuencia en la

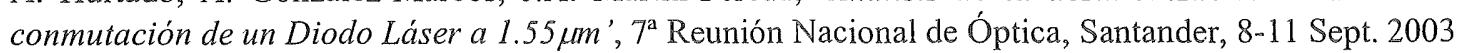

5. Ana González-Marcos, José A. Martín-Pereda. "Method to analyze the influence of hysteresis in optical arithmetic unit", Optical Engineering, , n40, pg. 2371-2385, (2001).

6. A. Hurtado, A. González-Marcos, J.A. Martín-Pereda, 'Optical Logic Cells with 1550 nm Laser Diode Amplifiers', unpublished.

7. S.V. Kartalopoulos, 'Introduction to DWDM Technology', IEEE Press Editioral Board, New York 1999. 